\title{
Estimation methods to extract complex permittivity from transmission coefficient in the terahertz band
}

\author{
Chuang Yang ${ }^{1,2,3,4} \cdot$ Jian Wang ${ }^{1,2}$ (D) Cheng Yang ${ }^{1,2}$
}

Received: 23 January 2021 / Accepted: 16 June 2021 / Published online: 22 July 2021

(c) The Author(s) 2021

\begin{abstract}
In this paper, the characteristics of the transmission coefficient $\left(S_{21}\right)$ measured in free-space at terahertz frequencies are analyzed. The analysis results are used to estimate the permittivity of the material under test, and the estimated permittivity is adopted as an initial-value for the iterative algorithm to extract the complex permittivity of the material from the $S_{21}$. The iterative extraction technique based on the estimation is efficient, while the iterative extraction technique without the estimation is inefficient. Various known materials in the literature are used to validate the technique in the terahertz band.
\end{abstract}

Keywords Complex permittivity $\cdot$ Terahertz $\cdot$ Free-space $\cdot$ Initial-guess

\section{Introduction}

Measurements of relative complex permittivity $\left(\varepsilon_{r}\right)$ in the terahertz $(\mathrm{THz})$ band are required for engineering designs of $\mathrm{THz}$ devices (e.g., lenses, waveguide, filters, etc.) (Hammler et al. 2016; Sahin et al. 2018a; Kazemipour et al. 2015; Yang et al. 2019; Güneşer 2019; Ali et al. 2017; Moradiani et al. 2020; Farmani et al. 2020). Free-space techniques have been widely used for dielectric characterization at $\mathrm{THz}$ frequencies, particularly since recent advances in $\mathrm{THz}$ components and instrumentation have made them more convenient and accurate (Zhang et al. 2017; Sahin et al. 2018b; Tosaka et al. 2015; Ghalichechian

Jian Wang

Wangjian16@tju.edu.cn

Chuang Yang

chuangyang@bupt.edu.cn

Cheng Yang

ych2041@tju.edu.cn

1 School of Microelectronics, Tianjin University, Tianjin 300072, China

2 Qingdao Institute for Ocean Technology of Tianjin University, Qingdao 266200, China

3 School of Information and Communication Engineering, Beijing University of Posts and Telecommunications, Beijing 100876, China

4 State Key Laboratory of Networking and Switching Technology, Beijing University of Posts and Telecommunications, Beijing 100876, China 
and Sertel 2015). The advances in the THz measurement can be summarized as two parts: (1) the gate-reflect-line (GRL) calibration technique and lens are used for the measurement system to improve the accuracy of the measurements (Zhang et al. 2017); (2) the environment of the calibration and the measurement is set to be clear to remove the impact of water vapor and $\mathrm{O} 2$ absorption lines (Sahin et al. 2018b), that also improve the accuracy of the measurements. However, the reflection coefficient $\left(\mathrm{S}_{11}\right)$ of the material under test (MUT) is still hard to be measured above $300 \mathrm{GHz}$ (Kazemipour et al. 2015; Lamb 1996). Since only the transmission coefficient $\left(S_{21}\right)$ can be used for the characterization above $300 \mathrm{GHz}$, the $\varepsilon_{r}$ of the MUT needs be extracted by the iterative algorithms.

But the iterative extraction techniques (e.g., Newtons method) restrict the application of the free-space transmission techniques, because the convergent values of the extracted $\varepsilon_{r}$ vary with the initial guesses for the iterative extraction techniques (see Fig. 5 in Zhang et al. 2017). Some publications simply ignored the problem on multiple convergent values (see Hammler et al. 2016; Sahin et al. 2018b; Tosaka et al. 2015), some experimentally reported the phenomena of getting multiple results and proposed method to obtain a right initial value by using $S_{11}$ and $S_{21}$ (Yang et al. 2019; Zhang et al. 2017), though $S_{11}$ is hard to be measured. While in Ghalichechian and Sertel (2015) technique without detailed analysis was proposed to estimate permittivity from $S_{21}$ in Zhang et al. (2017). But the technique fails to work when the electrical length of the MUT is large. In addition, the empirical value at low frequency of the MUT was adopted as the initial value for the iterative techniques to extract the $\varepsilon_{r}$ in the THz band (Ghalichechian and Sertel 2015). If there is a new material or the value of the $\varepsilon_{r}$ of the MUT in the THz band is much different from the value at low frequencies (see Fig. 10 in Seiler and Plettemeier (2019)). The extracted value of the permittivity is incorrect if the estimated value is far away from the true value (Yang et al. 2019). How to get a right initial-guess for the iterative extraction techniques is still an open-question.

In this paper, the proper initial-value for the iterative extraction techniques is estimated by using the properties of the measured $S_{21}$ in the THz band. The availability of the proposed technique is verified by various known materials (e.g. PMMA, PVC, Teflon, Glass, Crosslinked SUEX, etc.) in the THz-band.

\section{Theory and technique}

A schematic of a MUT with thickness of $t$ is shown in Fig.1. The MUT is measured in freespace. According to the analytical multilayer transmission model, transmission through a dielectric slab with thickness $t$ can be expressed as Farmani et al. (2020)

$$
S_{21}=\frac{4 \sqrt{\varepsilon_{r}} e^{-j \omega \sqrt{\mu_{0} \varepsilon_{0}} \sqrt{\varepsilon_{r} t}}}{\left(1+\sqrt{\varepsilon_{r}}\right)^{2}-\left(1-\sqrt{\varepsilon_{r}}\right)^{2} e^{-j 2 \omega \sqrt{\mu_{0} \varepsilon_{0}} \sqrt{\varepsilon_{r}} t}}
$$

where $\varepsilon_{r}=\varepsilon_{r}^{\prime}-j \varepsilon_{r}^{\prime \prime}$ is the relative complex permittivity of the MUT, $\omega$ is the angular frequency, and $t$ is outside of the square.

If the thickness of the MUT is larger than one or more wavelengths, the $S_{21}$ changes periodically with the frequency (Hammler et al. 2016). In the $\mathrm{THz}$ band, the wavelengths of the electromagnetic waves are short that the thickness of the MUT can be longer than one or more wavelengths. In this work, we estimate the permittivity of the MUT from the $S_{21}$ by using the special properties. 


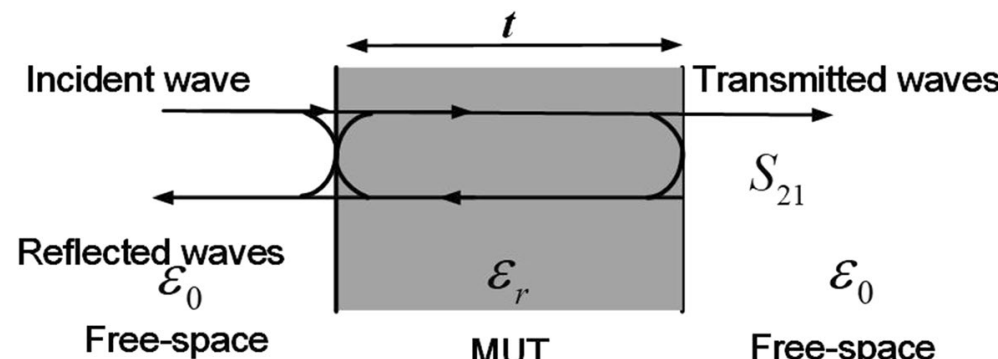

Fig. 1 A schematic of a MUT measured in free-space

\subsection{Method 1: using properties of $\left|S_{21}\right|$}

For low-loss materials, when the thickness of the MUT is an integer multiple of halfwavelength $(\lambda / 2)$ inside the MUT, there is a sharp peak in the measured $\left|S_{21}\right|$ due to Fabry CPerot effects (Ghalichechian and Sertel 2015). When there are two peaks in the measured $\left|S_{21}\right|$ and the MUT is non-dispersive or weakly dispersive between the two peak frequencies, the phase constants at the two frequencies can be derived as

$$
\begin{gathered}
\beta_{1}=n \pi / t \\
\beta_{2}=(n+1) \pi / t
\end{gathered}
$$

where $n$ is a positive integer.

As $(\alpha+j \beta)^{2}=-\omega^{2} \mu_{0} \varepsilon_{0}\left(\varepsilon_{r}^{\prime}-j \varepsilon_{r}^{\prime \prime}\right)$ and loss constant $\alpha$ is approximately equal to zero for low-loss materials, we can derive

$$
\beta^{2} \approx \omega^{2} \mu_{0} \varepsilon_{0} \varepsilon_{r}^{\prime}
$$

Combining Eqs. (2)-(4), the permittivity between the two peak frequencies of the MUT can be estimated as

$$
\varepsilon_{r}^{\prime} \approx\left(\frac{1}{2 t \sqrt{\varepsilon_{0} \mu_{0}} \Delta f_{1}}\right)^{2}
$$

where $\Delta f_{1}$ is the difference between the two peak frequencies.

\subsection{Method 2: using properties of $S_{21}$}

The method 1 is efficient when there are two or more peaks in the $\left|S_{21}\right|$. However, the peaks are not obvious when the MUT is thick as shown in Sahin et al. (2019). Because, the loss increases with the thickness, the resonance decreases with the loss. The difference between the peak and regular values is small. Therefore, we cannot use Eq. (5) for $\varepsilon_{r}^{\prime}$ estimation when the MUT is thick. 
For the thick MUT, there are more frequencies where $t$ is an integer multiple of wavelength inside the MUT. For the MUT with low loss tangent, the Eq. (1) can be approximately expressed as

$$
S_{21} \approx \frac{4 \sqrt{\varepsilon_{r}^{\prime}} e^{-j \omega \sqrt{\mu_{0} \varepsilon_{0}} \sqrt{\varepsilon_{r}^{\prime} t}}}{\left(1+\sqrt{\varepsilon_{r}^{\prime}}\right)^{2}-\left(1-\sqrt{\varepsilon_{r}^{\prime}}\right)^{2} e^{-j 2 \omega \sqrt{\mu_{0} \varepsilon_{0}} \sqrt{\varepsilon_{r}^{\prime} t}}}
$$

As seen in Eq. (6), the $\angle S_{21}$ is approximately $180^{\circ}$ when the thickness of the MUT is an integer multiple of wavelength inside the MUT. We processed the two $\left|S_{21}\right|$ at two frequencies where $\angle S_{21}$ are equal $180^{\circ}$, the permittivity of the MUT can be estimated as

$$
\varepsilon_{r}^{\prime} \approx\left(\frac{1}{t \sqrt{\varepsilon_{0} \mu_{0}} \Delta f_{2}}\right)^{2}
$$

where $\Delta f_{2}$ is the difference between the two frequencies.

\subsection{Application procedures of the two methods}

The application procedures of the proposed methods are shown as follows:

Step 1: choose the method according to the characteristics of the $S_{21}$. If the number of the peaks in the $\left|S_{21}\right|$ is more than the number of the frequencies where the values of $\angle S_{21}$ are equal to $180^{\circ}$, the method 1 is chosen. Otherwise, the method 2 is chosen.

Step 2: determine the dispersion characteristics of the MUT. If all $\Delta f_{1}$ (or $\Delta f_{2}$ ) are approximate for the MUT, the MUT is non-dispersive over the entire measured frequency range. Otherwise, the MUT is dispersive over the entire measured frequency range.

Step 3.1: for the MUT which is non-dispersive over the entire measured frequency range, the average value of the $\Delta f_{1}$ (or $\Delta f_{2}$ ) is used for the permittivity estimation.

Step 3.2: for the MUT which is dispersive over the entire measured frequency range, the value of the $\Delta f_{1}$ (or $\Delta f_{2}$ ) is used for the permittivity estimation between the two adjacent frequencies. We can see that $\Delta f$ would be used for different frequency band. If the materials whose permittivity change dramatically with frequency are measured, we should make them thick enough, that the permittivity between the $\Delta f$ changes a little. Then the proposed can be efficient.

Step 4: Newtons method with the estimated initial value (permittivity) is performed.

\section{Examples and discussion}

\subsection{The non- or weak dispersive samples in the literature}

To validate the proposed technique, the $\left|S_{21}\right|$ in the figures of Hammler et al. (2016), Kazemipour et al. (2015), Sahin et al. (2018b) and Bourreau et al. (2006) are used for determining the $\Delta f$. We obtained the peak frequencies of the $\left|S_{21}\right|$ and processed the frequencies by Eq. (4). In theoretical, the values of the $\left|S_{21}\right|$ and the $\left|S_{21}\right|$ are equal to each other. The permittivity calculated by Eq. (5) agrees well with the extracted values in the literature as show in Table 1. The error between the calculated values and the values in the 


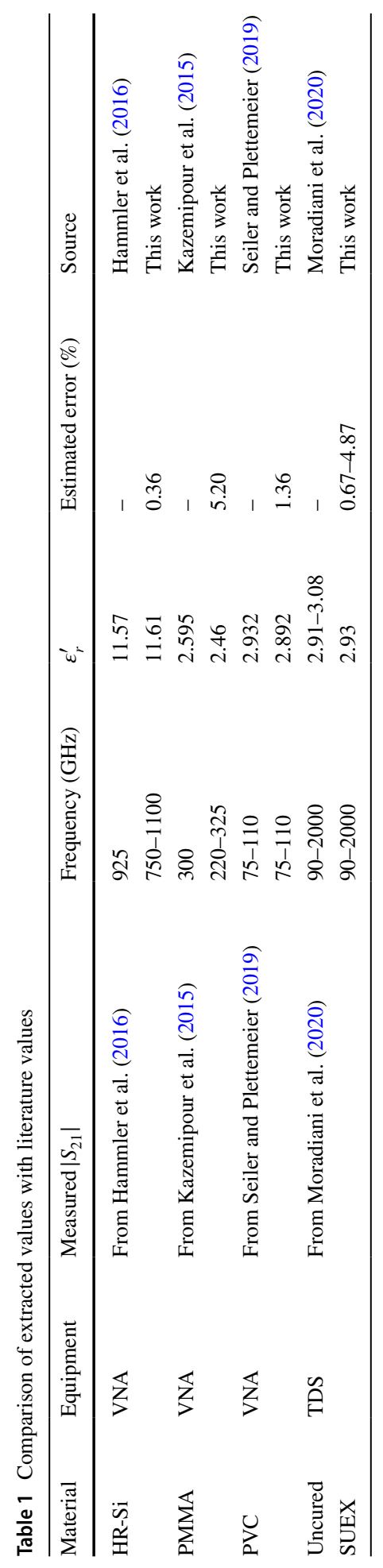


literature ranges from 0.36 to $5.20 \%$. However, the phase angles of the $S_{21}$ are not presented in Kazemipour et al. (2015), Sahin et al. (2018b) and Bourreau et al. (2006). We cannot determine the $\varepsilon_{r}$ over the entire measured frequency range by using the Newtons method.

In this work, we used the extracted in Kazemipour et al. (2015) and Sahin et al. (2018b) to simulate the $S_{21}$ in the THz band. The $\varepsilon_{r}$ of the materials are put into Eq. (1). The simulation method is shown in Seiler and Plettemeier (2019). The simulated S-parameters are calculated using the values of $\varepsilon_{r}$ (Bourreau et al. 2006). That is reasonable, because the measured and simulated S21 in free-space at $\mathrm{THz}$ frequencies are almost the same (see Fig. 6 in Bourreau et al. (2006)). In our previous work, $S$-parameters simulated in the THz band have been further proved for validating extraction techniques (Yang et al. 2019).

Since the measurable frequency range is different when using a vector network analyzer (VNA) and by TDS (time-domain spectroscopy) (Tosaka et al. 2015), PMMA, PVC, Teflon, Plexiglas (a kind of Glass) with various thicknesses were simulated between 260 and $400 \mathrm{GHz}$ (VNA measurement THz band) and the Crosslinked SUEX with various thicknesses were simulated between 100 and $2000 \mathrm{GHz}$ (TDS measurement $\mathrm{THz}$ band). As shown in Fig. 2, the $S_{21}$ of the PMMA is presented as an example to show that the S21 can be analyzed by the methods in Sect. 2 .

As shown in Table 2, we estimated the $\varepsilon_{r}^{\prime}$ of these materials by using Eqs. (5) and (7). The estimated values approximate to the true values. It is found that the equations may not be applicable simultaneously in some cases. For example, the $\varepsilon_{r}^{\prime}$ of the $8 \mathrm{~mm}$ PMMA needs to be estimated by Eq. (5) as the peaks are not obvious for the $\left|S_{21}\right|$ of the PMMA, and the $\varepsilon_{r}^{\prime}$ of the $2 \mathrm{~mm}$ PVC needs to be estimated by Eq. (5) as the thickness of the PVC is less than a wavelength. Thus, the two proposed methods for $\varepsilon_{r}^{\prime}$ estimation should be used simultaneously. After estimation, the estimated $\varepsilon_{r}^{\prime}$ is adopted as the initial-value for the Newtons method. The tolerance of the algorithm is set to be 10e-7 according to Zhang et al. (2017). For each frequency, it takes about 0.14 second to converge using the initial value estimated by the proposed.

The extracted values of the Teflon, PVC, glass and crosslinked SUEX agree well with the true values as shown in Figs. 3 and 4. That further validates the proposed methods.

\subsection{The dispersive sample in the literature}

Those samples in the Part A have minimal variation in $\varepsilon_{r}$ from low to high frequencies. To further show the excellent performance of the proposed, a dispersive sample
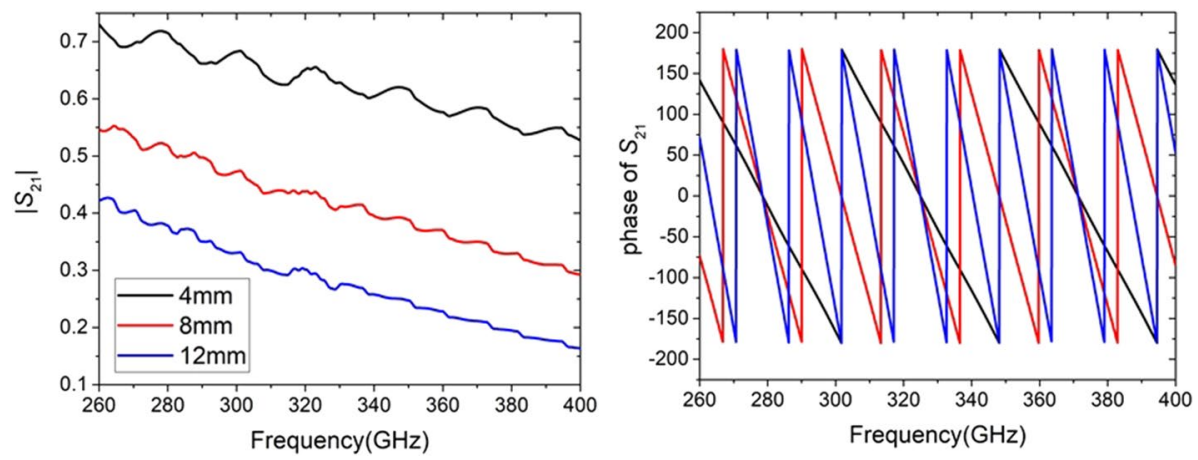

Fig. $2 S_{21}$ of three PMMA samples with different thickness 
Table 2 Estimated initial values $\left(\varepsilon_{r}^{\prime}\right)$ for the iterative algorithm

\begin{tabular}{|c|c|c|c|c|c|c|}
\hline \multirow[t]{2}{*}{ Material } & \multirow{2}{*}{$\begin{array}{l}\text { Thickness } \\
(\mathrm{mm})\end{array}$} & \multirow{2}{*}{$\begin{array}{l}\text { Frequency } \\
(\mathrm{GHz})\end{array}$} & \multicolumn{2}{|c|}{ Method 1 (F-P resonance) } & \multicolumn{2}{|c|}{ Method $2\left(\angle S_{21}=180^{\circ}\right)$} \\
\hline & & & $\Delta f_{1}(\mathrm{GHz})$ & $\begin{array}{l}\text { Estimated } \\
\text { value }\end{array}$ & $\Delta f_{2}(\mathrm{GHz})$ & Estimated value \\
\hline \multirow[t]{3}{*}{ PMMA } & 4 & $260-400$ & 23.04 & 2.6491 & 46.4 & 2.6127 \\
\hline & 8 & & - & - & 23.2 & 2.6127 \\
\hline & 12 & & - & - & 15.46 & 2.6141 \\
\hline \multirow[t]{3}{*}{ Glass } & 2 & & 29.45 & 6.4812 & 59.45 & 6.3661 \\
\hline & 4 & & - & - & 29.67 & 6.3898 \\
\hline & 6 & & - & - & 19.8 & 6.3769 \\
\hline \multirow[t]{3}{*}{ PVC } & 2 & & 45.75 & 2.6874 & - & - \\
\hline & 4 & & 29.12 & 2.9267 & 44.85 & 2.7964 \\
\hline & 8 & & - & - & 22.38 & 2.8076 \\
\hline \multirow[t]{3}{*}{ Teflon } & 2 & & 51.7 & 2.1045 & - & - \\
\hline & 8 & & 12.93 & 2.1028 & 25.88 & 2.0996 \\
\hline & 16 & & 6.47 & 2.0995 & 12.93 & 2.1028 \\
\hline Crosslinked & 0.15 & $100-2000$ & 617.5 & 2.6226 & 1247 & 2.5723 \\
\hline \multirow[t]{2}{*}{ SUEX } & 0.45 & & 205.25 & 2.6375 & 410.75 & 2.6342 \\
\hline & 1 & & - & - & 185.89 & 2.6046 \\
\hline
\end{tabular}
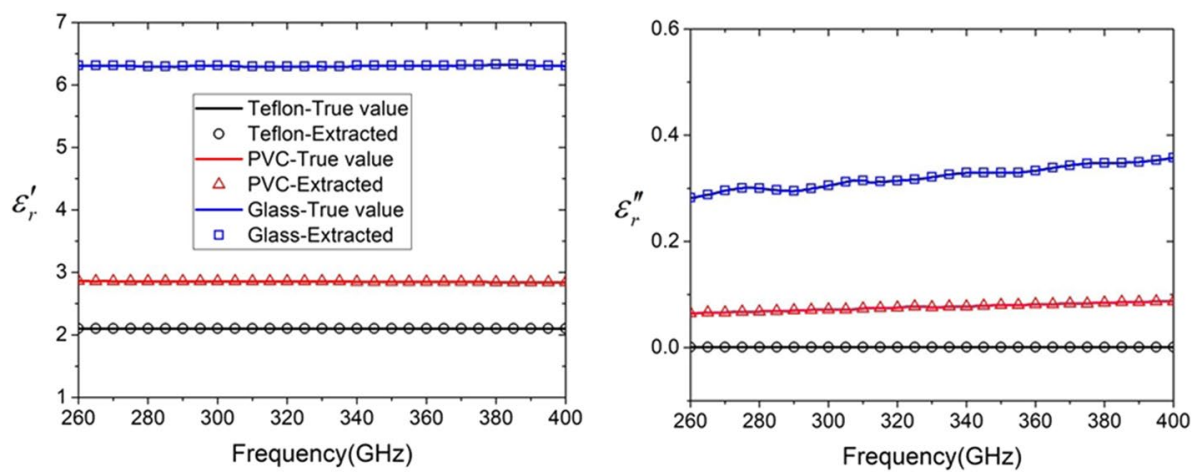

Fig. 3 Extracted complex permittivity of $16 \mathrm{~mm}$ Teflon, $8 \mathrm{~mm}$ PVC and $6 \mathrm{~mm}$ glass samples

Benzocyclobutene (BCB) with thickness of $2 \mathrm{~mm}$ is used for validation. The $\varepsilon_{r}^{\prime}$ is obtained from Seiler and Plettemeier (2019). The permittivity of the BCB is about 5.5 at $0.5 \mathrm{GHz}$, and is about 2.6 in the $\mathrm{THz}$ band as shown in Fig. 5. The permittivity of the $\mathrm{BCB}$ at low frequency is twice than that at the terahertz frequency.

As shown in Fig. 6, the extracted $\varepsilon_{r}^{\prime}$ of the BCB is wrong when using the permittivity at $4,4.5$ and $5 \mathrm{GHz}$ as the initial values which are far away from the true values. And the values of the $\varepsilon_{r}^{\prime}$ extracted by the proposed method agree well with the true values. 
Fig. 4 Extracted complex permittivity of $1 \mathrm{~mm}$ crosslinked SUEX

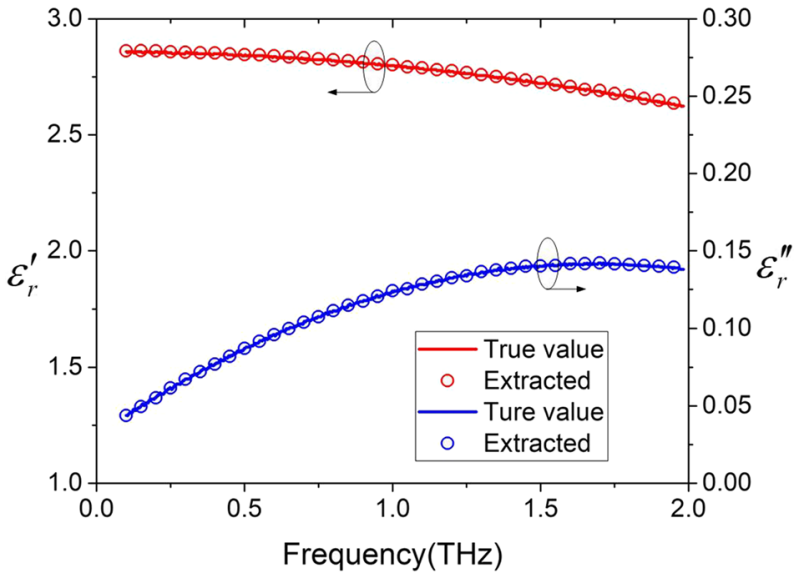

Fig. 5 Permittivity of $\mathrm{BCB}$ [obtained from Fig. 10 in Seiler and Plettemeier (2019)]
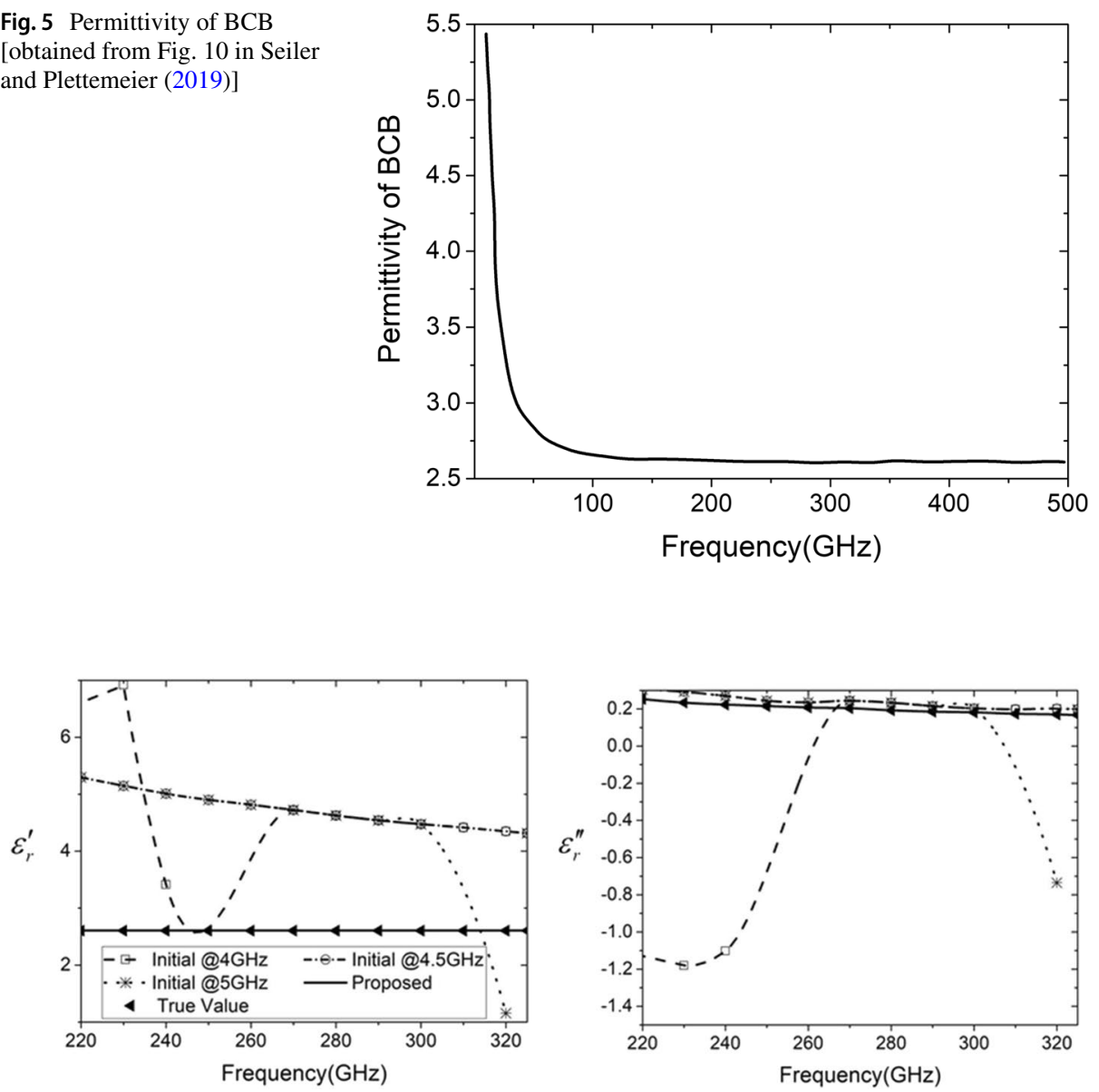

Fig. 6 Extracted complex permittivity of $2 \mathrm{~mm}$ BCB with different initial values from 220 to $330 \mathrm{GHz}$ 


\section{Conclusions}

In this work, a technique is proposed to estimate the permittivity of the MUT from the measured $S_{21}$. The estimated permittivity is a correct initial-value for the iterative technique which extracts the $\varepsilon_{r}$ from the $S_{21}$. Since only the $S_{21}$ can be efficiently measured in the $\mathrm{THz}$ band, the proposed is important for material characterization at $\mathrm{THz}$ frequencies. The proposed technique is efficient when the measured $\left|S_{21}\right|$ have peak values at multiple frequencies or the measured values of the $\angle S_{21}$ are equal to $180^{\circ}$ at multiple frequencies.

Funding Information This research was funded by the National Natural Science Foundation of China, Grant Number 62031008.

Open Access This article is licensed under a Creative Commons Attribution 4.0 International License, which permits use, sharing, adaptation, distribution and reproduction in any medium or format, as long as you give appropriate credit to the original author(s) and the source, provide a link to the Creative Commons licence, and indicate if changes were made. The images or other third party material in this article are included in the article's Creative Commons licence, unless indicated otherwise in a credit line to the material. If material is not included in the article's Creative Commons licence and your intended use is not permitted by statutory regulation or exceeds the permitted use, you will need to obtain permission directly from the copyright holder. To view a copy of this licence, visit http://creativecommons.org/licenses/by/4.0/.

\section{References}

Ali, F., Mehdi, M., Sheikhi, M.H.: Tunable resonant Goos-Hnchen and Imbert-Fedorov shifts in total reflection of terahertz beams from graphene plasmonic metasurfaces. J. Opt. Soc. Am. B 34, 10971106 (2017). https://doi.org/10.1364/JOSAB.34.001097

Bourreau, D., Peden, A., Maguer, S.L.: A quasi-optical free-space measurement setup without time-domain gating for material characterization in the W-band. IEEE Trans. Instrum. Meas. 55, 2022-2028 (2006). https://doi.org/10.1109/TIM.2006.884283

Farmani, H., Farmani, A.: Graphene sensing nanostructure for exact graphene layers identification at terahertz frequency. Physica E: Low-dimensional Systems and Nanostructures 124, 1-5 (2020). https://doi. org/10.1016/j.physe.2020.114375

Ghalichechian, N., Sertel, K.: Permittivity and loss characterization of SU-8films for mmW and terahertz applications. IEEE Antennas Wireless Propag. Lett. 14, 723-726 (2015). https://doi.org/10.1109/ LAWP.2014.2380813

Güneşer, M.T.: Artificial intelligence solution to extract the dielectric properties of materials at sub-THz frequencies IET Sci. Meas. Technol. 13, 523-528 (2019). https://doi.org/10.1049/iet-smt.2018.5356

Hammler, J., Andrew, J.G., Claudio, B.: Free-space permittivity measurement at terahertz frequencies with a vector network analyzer. IEEE Trans. THz Sci. Technol. 6, 817-823 (2016). https://doi.org/10.1109/ TTHZ.2016.2609204

Kazemipour, A., Hudlička, M., Yee, S., Salhi, M., Kleine-Ostmann, T., Schrader, T.: Design and calibration of a compact quasi-optical system for material characterization in millimeter/submillimeter wave domain. IEEE Trans. Instrum. Meas. 64, 1438-1445 (2015). https://doi.org/10.1109/TIM.2014.23761 15

Lamb, W.J.: Miscellaneous data on materials for millimetre and submillimetre optics. Int. J. Infrared Millimeter Waves 17, 1997-2034 (1996). https://doi.org/10.1007/BF02069487

Moradiani, F., Farmani, A., Yavarian, M., Mir, A., Behzadfar, F.: A multimode graphene plasmonic perfect absorber at terahertz frequencies. Phys. E Low-Dimens. Syst. Nanostruct. 122, 1-7 (2020). https://doi. org/10.1016/j.physe.2020.114159

Sahin, S., Nahar, N.K., Sertel, K.: Thin-film SUEX as an anti-reflection coating for mmW and THz applications. IEEE Trans. THz Sci. Technol. 9, 417-421 (2018). https://doi.org/10.1109/TTHZ.2019.2915672

Sahin, S., Nahar, N.K., Sertel, K.: Permittivity and loss characterization of SUEX epoxy films for mmW and THz applications. IEEE Trans. THz Sci. Technol. 8, 397-402 (2018). https://doi.org/10.1109/TTHZ. 2018.2840518 
Sahin, S., Nahar, N.K., Sertel, K.: Dielectric properties of low-loss polymers for mmW and THz applications. J. Infrared Millim. THz Wave 40, 557-573 (2019). https://doi.org/10.1007/s10762-019-00584-2

Seiler, P., Plettemeier, D.: A method for substrate permittivity and dielectric loss characterization up to subterahertz frequencies. IEEE Trans. Microw. Theory Techn. 64, 1640-1651 (2019). https://doi.org/10. 1109/TMTT.2019.2897102

Tosaka, T., Fujii, K., Fukunaga, K., Kasamatsu, A.: Development of complex relative permittivity measurement system based on free-space in 220-330-GHz range. IEEE Trans. THz Sci. Technol. 5, 102-109 (2015). https://doi.org/10.1109/TTHZ.2014.2362013

Yang, C., Ma, K., Ma, J.-G.: A noniterative and efficient technique to extract complex permittivity of lowloss dielectric materials at terahertz frequencies. IEEE Antennas Wirel. Propag. Lett. 18, 1971-1975 (2019). https://doi.org/10.1109/LAWP.2019.2935170

Zhang, X., Chang, T., Cui, H.-L., Sun, Z., Yang, C.: A free-space measurement technique of terahertz dielectric properties. J. Infrared Millim. THz Waves 38(3), 356-365 (2017). https://doi.org/10.1007/ s10762-016-0341-2

Publisher's Note Springer Nature remains neutral with regard to jurisdictional claims in published maps and institutional affiliations. 